\title{
Two-step synthesis of fluorescent 3-arylated 1,3a,6a-triazapentalenes via a three-component triazolization reaction
}

\author{
Bram Verbelen and Wim Dehaen* \\ Department of Chemistry, KU Leuven, Celestijnenlaan 200f - bus 02404, 3001 Leuven, Belgium. \\ Supporting Information Placeholder
}

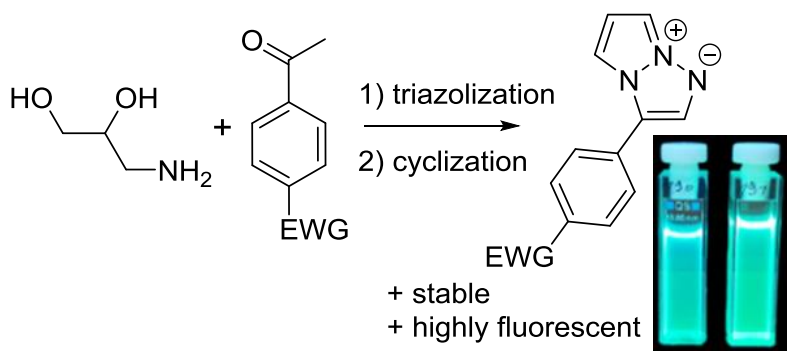

\begin{abstract}
Novel 3-arylated 1,3a,6a-triazapentalenes were synthesized using a metal-free three-component triazolization reaction followed by a triflate based cyclization step. This method starts from simple commercial starting materials and provides an easy functionalization strategy for the 3-position of the triazapentalenes. By introducing electron withdrawing groups onto this position, stable and highly fluorescent dyes could be synthesized.
\end{abstract}

Small fluorescent organic molecules are indispensable tools in many applications as biological labels and probes, chemosensors, laser dyes, photo-active materials in light-emitting devices, etc. ${ }^{1}$ Recently, 1,3a,6a-triazapentalenes $\mathbf{3}$ were discovered as compact $10 \pi$-electron fluorophores exhibiting various promising properties, such as emission in the UV to visible light region between $380 \mathrm{~nm}$ and $630 \mathrm{~nm}$, a strong solvatochromism, a large Stokes-shift, solubility in water and a very small size., Unfortunately, most triazapentalenes $\mathbf{3}$ exhibited modest to poor stability. This low stability has been explained as either susceptibility to air oxidation ${ }^{4}$ in analogy with 3a,6a-diazapentalenes, ${ }^{5}$ to protonation ${ }^{2 \mathrm{a}}$ or to dimerization ${ }^{3}$ in analogy with pentalenes. ${ }^{6}$ However, introducing an electron withdrawing group on the triazapentalene core can improve the stability of the resulting dye. ${ }^{3}$ Indeed, the majority of the reported 1,3a,6a-triazapentalenes $\mathbf{3}$ bear an electron withdrawing group at their 2- and/or 3-positions., ${ }^{2,3}$
Currently, only two synthetic pathways towards fluorescent triazapentalenes have been reported. ${ }^{2,3}$ The first published procedure is based on a copper-catalyzed azide-alkyne cycloaddition between a 3-azidopropane-1,2-diol bis(trifluoromethanesulfonate) $\mathbf{1}$ and a terminal alkyne $\mathbf{2}$ (Scheme 1, path A). ${ }^{2}$ Under the basic reaction conditions an intramolecular substitution followed by base-induced elimination and aromatization takes place to form the desired 1,3a,6a-triazapentalene 3 . Unfortunately, this method is only useful to synthesize 2-substituted fluorophores. The other positions are much more difficult to functionalize using this strategy, ${ }^{2 b, e}$ whereas the 3-position cannot be functionalized because this reaction would require an internal alkyne which is unreactive under click reaction conditions.

Scheme 1. Summary of the synthetic pathways towards fluorescent triazapentalenes and the numbering of the triazapentalene core
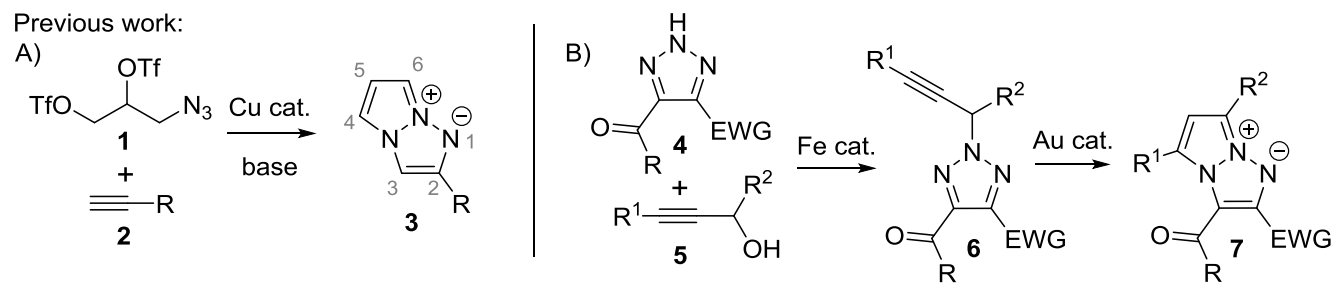

This work:

$$
\text { C) }
$$<smiles>NCC(O)CO</smiles><smiles>[R]C(C)=O</smiles><smiles>CO[Mg]O[Na]</smiles>

HO 9

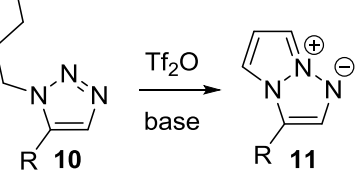


The second reported pathway towards triazapentalenes starts with an iron-catalyzed alkylation of a $2 \mathrm{H}-1,2,3$-triazole 4 with a propargyl alcohol $\mathbf{5}$ followed by a gold-catalyzed cyclization (Scheme 1, path B). ${ }^{3}$ This method allows the introduction of substituents onto the 4- and 6-positions of the fluorophore. However, the starting triazole 4 requires electron withdrawing substituents to ensure the correct regiochemistry for the alkylation step and to allow the gold-catalyzed cyclization step. Hence, the 2,3-positions are not easy to modify with this strategy, particularly the scope of the 3-position, which was always substituted with a carbonyl functional group, is limited. An additional disadvantage of both procedures is that their starting materials, 3-azidopropane-1,2-diol bis(trifluoromethanesulfonate) $\mathbf{1}$ for the first protocol and $2 \mathrm{H}$-1,2,3-triazole $\mathbf{4}$ and propargyl alcohol $\mathbf{5}$ for the second procedure, are not commercially available.

Recently, our group developed a metal-free three-component reaction between a primary amine, an enolizable carbonyl compound and $p$-nitrophenyl azide to synthesize 1,4,5-trisubstituted 1,2,3-triazoles. ${ }^{7}$ We hypothesized that by using the commercially available 3-aminopropane-1,2-diol $\mathbf{8}$ as the primary amine it should be possible to make with this reaction a triazole 10 containing a 2,3-dihydroxypropyl at its 1-position (Scheme 1 , path C). Such a triazole could be a precursor for the synthesis of a 3-functionalized 1,3a,6a-triazapentalene 11, whose accessibility is still very limited using the current procedures, by transforming the hydroxyl groups into leaving groups so that under the influence of base the desired pyrazolium ring can be formed by substitution and elimination (Scheme 1, path $\mathrm{C}$ ). Hence, we set out to investigate the feasibility of this novel strategy to synthesize new 3-arylated triazapentalenes 11. Furthermore, the spectroscopic properties of these new dyes were measured in detail.

Table 1. Synthesis of the triazapentalene precursor triazole $\mathbf{1 0}^{\mathrm{a}}$

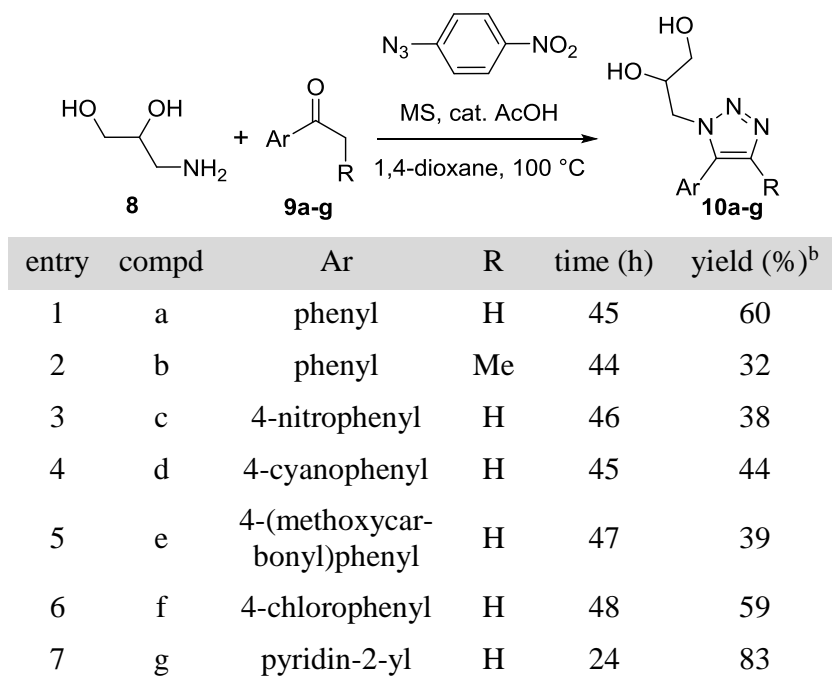

${ }^{\text {a }}$ Experimental conditions: $0.5 \mathrm{mmol}$ aryl ketone 9, 1.1 equivalents $p$-nitrophenyl azide, 1.2 equivalents 3-aminopropane-1,2diol 8, $60 \mathrm{mg} 4 \AA$ molecular sieves (MS), $30 \mathrm{~mol} \% \mathrm{AcOH}, 2.5$ $\mathrm{mL}$ 1,4-dioxane, stirring for the indicated time at $100{ }^{\circ} \mathrm{C}$. ${ }^{\mathrm{b}}$ Isolated yield.

In order to synthesize the precursor 10, the three-component reaction developed in our group was used. ${ }^{7}$ For the initial test reaction 3-aminopropane-1,2-diol 8 was reacted with acetophenone 9a and $p$-nitrophenyl azide using 4 Å molecular sieves and a catalytic amount of acetic acid in toluene at $100{ }^{\circ} \mathrm{C}$. Unfortunately, 3-aminopropane-1,2-diol $\mathbf{8}$ did not dissolve in toluene under the used reaction conditions and only a trace amount of the desired triazole 10a was formed. However, when the more polar 1,4-dioxane was used as the solvent the amine did dissolve and reaction occurred, producing the expected 5-phenyl-1,2,3triazole 10a in a yield of $60 \%$ (Table 1, entry 1 ).

Similarly, using propiophenone $\mathbf{9 b}$ as the aryl ketone resulted in a 4-methyl-5-phenyl triazole 10b (Table 1, entry 2). Moreover, substituted acetophenones 9c-f under the same reaction conditions gave the corresponding 5-arylated triazoles 10c-f in modest yields (Table 1, entries 3-6). Reaction with 2-acetylpyridine $9 \mathrm{~g}$ was faster than the other reactions and produced a 5(pyridin-2-yl) triazole $\mathbf{1 0 g}$ in an excellent yield of $83 \%$ (Table 1, entry 7).

Table 2. Synthesis of 1,3a,6a-triazapentalenes 11 starting from substituted triazoles $\mathbf{1 0}^{\mathrm{a}}$

$\begin{array}{cccccc}\text { entry } & \text { compd } & \mathrm{Ar} & \mathrm{R} & \text { time (min) } & \text { yield (\%) } \\ 1 & \mathrm{a} & \text { phenyl } & \mathrm{H} & 30 & \text {-c }^{\mathrm{b}} \\ 2 & \mathrm{~b} & \text { phenyl } & \mathrm{Me} & 30 & \text {-c }^{\mathrm{c}} \\ 3 & \mathrm{c} & \text { 4-nitrophenyl } & \mathrm{H} & 40 & 69 \\ 4 & \mathrm{~d} & \text { 4-cyanophenyl } & \mathrm{H} & 30 & 76 \\ 5 & \mathrm{e} & \text { 4-(methoxycar- } & \mathrm{H} & 30 & 70 \\ 6 & \mathrm{f} & \text { 4-chlorophenyl } & \mathrm{H} & 30 & \text {-c }^{\mathrm{c}} \\ 7 & \mathrm{~g} & \text { pyridin-2-yl } & \mathrm{H} & 80 & \text {-c }^{\mathrm{c}}\end{array}$

${ }^{\text {a }}$ Experimental conditions: $0.1 \mathrm{mmol}$ triazole 10, 10 equivalents pyridine, 3 equivalents $\mathrm{Tf}_{2} \mathrm{O}, 1 \mathrm{~mL}$ DCM, stirring for the indicated time at $0{ }^{\circ} \mathrm{C}$, afterwards the reaction was quenched by addition of 6 equivalents of $\mathrm{MeOH} .{ }^{b}$ Isolated yield. ${ }^{\mathrm{c}}$ Product decomposes during work up.

To obtain the desired triazapentalene compounds $\mathbf{1 1}$ the hydroxyl groups of the triazoles $\mathbf{1 0}$ need to be transformed into leaving groups so that in the presence of base the pyrazolium ring of the final products $\mathbf{1 1}$ can be formed. The first published procedure to make fluorescent triazapentalenes used triflates as leaving groups for a similar cyclization step. ${ }^{2 a}$ The cyclization was unsuccessful, however, when a mesylate or a tosylate was used instead of a triflate. ${ }^{2 a}$ Even though our strategy is different for those in literature, triazole $\mathbf{1 0}$ containing triflate substituents might be able to ring close to a 1,3a,6a-triazapentalene. Hence, to test the cyclization step with our triazoles 10, the hydroxyl groups were converted into triflate leaving groups. To this end, 5-phenyl triazole 10a was reacted under standard triflate synthesis conditions, ${ }^{8}$ using triflic anhydride and pyridine in dichloromethane at $0{ }^{\circ} \mathrm{C}$. This resulted in a quick consumption of the starting material 10a and the formation of a new product. Unfortunately, attempts to isolate this new product were unsuccessful as this product decomposed during work up (Table 2, entry 1). Most of this decomposition occurred during evaporation resulting in several unidentifiable colored compounds. However, the NMR of this crude dark green-blue mixture did 
show that it contained the desired 3-phenyl triazapentalene dye 11a (Figure S1 and S2). Mass spectroscopy of this crude mixture also confirmed the presence of the desired compound 11a (Figure S3). Sadly, the instability of this triazapentalene 11a prevented its isolation and full characterization. When 5-phenyl triazole 10a was converted to a bistosylate instead of a bistriflate, again no cyclization occurred under the influence of base, not even when the reaction mixture was heated.

In an attempt to limit the decomposition of the final product, the more substituted 4-methyl-5-phenyl triazole 10b was reacted with triflic anhydride under the same conditions. Unfortunately, the formed product also decomposed during work up and no pure compound could be obtained (Table 2, entry 2). Nonetheless, crude NMR (Figure S4) and mass spectroscopy (Figure S5) showed that the expected 2-methyl-3-phenyltriazapentalene 11b was indeed formed during the reaction.

It has been mentioned in the literature that placing an electron withdrawing group onto the triazapentalene core can improve the stability of the resulting dye. ${ }^{2 a, 3}$ Hence, a series of triazoles 10c-g bearing electron poor aryl groups were subjected to the cyclization reaction, in order to test if they could form more stable triazapentalene derivatives 11c-g (Table 2, entries 3-7). Reacting triazoles with strongly electron withdrawing 4-nitrophenyl (in compound 10c), 4-cyanophenyl (in compound 10d) or 4-(methoxycarbonyl)phenyl (in compound 10e) substituents did indeed produce stable 3-aryl-triazapentalene dyes 11c-e that could be isolated in good yields of around $70 \%$ (Table 2, entries 3-5). The less electron withdrawing 4-chlorophenyl (in triazole 10f) or pyridin-2-yl (in triazole $\mathbf{1 0 g}$ ) groups were unfortunately not stabilizing enough and the corresponding triazapentalenes 11f-g decomposed during work up and could not be isolated (Table 2, entries 6 and 7).

The three reactions that gave the stable 1,3a,6atriazapentalene dyes 11c-e only formed this compound after quenching the reaction with methanol at room temperature. After addition of triflic anhydride at $0{ }^{\circ} \mathrm{C}$ and consumption of the starting material 10c-e but before quenching the reaction, the reaction mixture contained a different product, which is assumed to be the bistriflate intermediate. Nonetheless, after quenching and stirring at room temperature a colored compound was formed that could be isolated. Characterization proved that this colored compound is indeed the desired triazapentalene dye 11c-e.

The spectroscopic properties of the newly synthesized 3-aryltriazapentalene dyes 11c-d (Figure 1) were studied in three solvents (Table 3, Figure S6, Figure S7, Figure S8). As these new compounds seem to be stable under UV irradiation, in contrast to most reported triazapentalenes, ${ }^{2}$ their absorption and emission spectra and their fluorescence quantum yield could be fully determined. The results of this investigation are presented in Table 3.
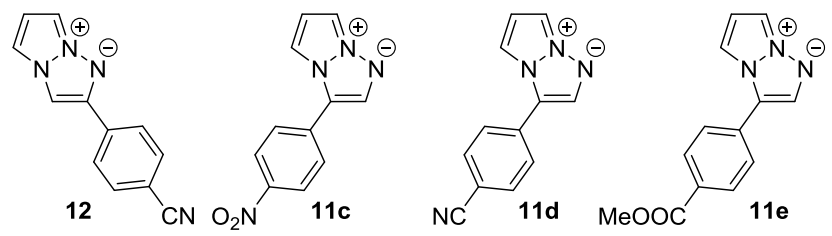

Figure 1. Structures of the new 3-aryl-triazapentalene dyes 11c-e for which the spectroscopic properties were measured together with the structure of the reference compound 12 . $^{2 a}$

For comparison, the spectroscopic properties of the related and previously reported ${ }^{2 a}$ 2-(4-cyanophenyl)-triazapentalene $\mathbf{1 2}$ were added to Table 3 as well. This allows us to compare the effect of 2-aryl and 3-aryl substituents on the characteristics of the resulting dyes. The absorption maximum of the dye with a 4-cyanophenyl substituent at the 3-position 11d is $20 \mathrm{~nm}$ red shifted compared to its 2-substituted derivative 12. In contrast, the emission maxima show the opposite trend, the 2-arylated dye 12 emits at a longer wavelength than the 3-functionalized derivative 11d. Hence, the Stokes shift of 2-(4-cyanophenyl)triazapentalene 12 is larger than the Stokes shift of 3-(4-cyanophenyl)-triazapentalene 11d.

Table 3. Spectroscopic data of the new 3-aryl-triazapentalene dyes 11c-d and the reference compound $\mathbf{1 2}^{2 \mathrm{a}}$ in three solvents

\begin{tabular}{|c|c|c|c|c|c|c|c|}
\hline dye & solvent ${ }^{\mathrm{a}}$ & $\lambda_{\text {abs, } \max }(\mathrm{nm})$ & $\lambda_{\mathrm{em}, \max }(\mathrm{nm})$ & Stokes shift $\left(\mathrm{cm}^{-1}\right)$ & fwhmabs $\left(\mathrm{cm}^{-1}\right)$ & fwhmem $\left(\mathrm{cm}^{-1}\right)$ & $\Phi_{\mathrm{f}}^{\mathrm{b}}$ \\
\hline \multirow[t]{3}{*}{$12^{2 \mathrm{a}}$} & $\mathrm{MeCN}$ & $-{ }^{c}$ & 548 & $-\mathrm{c}$ & $-{ }^{c}$ & $-c$ & 0.021 \\
\hline & DCM & 381 & 509 & 6600 & $-c$ & $-c$ & 0.18 \\
\hline & benzene & $-^{c}$ & 464 & $-{ }^{c}$ & $-{ }^{c}$ & $-^{c}$ & 0.17 \\
\hline \multirow[t]{3}{*}{$11 \mathrm{c}$} & $\mathrm{MeCN}$ & 471 & $-^{\mathrm{d}}$ & $-{ }^{\mathrm{d}}$ & 4542 & $-^{\mathrm{d}}$ & $-^{d}$ \\
\hline & DCM & 475 & $-^{\mathrm{d}}$ & $-\mathrm{d}$ & 4039 & $-\mathrm{d}$ & $-^{\mathrm{d}}$ \\
\hline & toluene & 470 & $-{ }^{\mathrm{e}}$ & $-\mathrm{e}$ & 3563 & $-\mathrm{e}$ & $-\mathrm{e}$ \\
\hline \multirow[t]{3}{*}{$11 \mathrm{~d}$} & $\mathrm{MeCN}$ & 397 & 504 & 5348 & 2916 & 4755 & 0.17 \\
\hline & DCM & 401 & 464 & 3386 & 2686 & 3495 & 0.57 \\
\hline & toluene & 403 & 442 & 2189 & 2829 & 2522 & 0.12 \\
\hline \multirow[t]{3}{*}{$11 \mathrm{e}$} & $\mathrm{MeCN}$ & 395 & 522 & 6159 & 3417 & 4418 & 0.04 \\
\hline & DCM & 399 & 476 & 4054 & 3267 & 3675 & 0.79 \\
\hline & toluene & 404 & 443 & 2179 & 2886 & 2597 & 0.29 \\
\hline
\end{tabular}

${ }^{a}$ Solvents are listed from top to bottom according to increasing refractive index $n .{ }^{\mathrm{b}}$ Fluorescence quantum yield determined vs fluorescein in ethanol $\left(\Phi_{\mathrm{r}}=0.79\right)$ as a reference. ${ }^{c}$ Data were not reported in the literature. ${ }^{2 \mathrm{~d} d}$ No fluorescence was detected.

${ }^{\mathrm{e}}$ Fluorescence was too weak to determine this value accurately. 
Comparing the different 3-aryl triazapentalenes, shows that the absorption maxima of the ester dye 11e are located at similar wavelengths as those of the cyano derivative 11d around 400 $\mathrm{nm}$. Furthermore, the absorption maxima of both compounds are slightly bathochromically shifted when going from polar to apolar solvents. In contrast, a more significant blue shift is observed in the emission maxima of both compounds, located between $442 \mathrm{~nm}$ and $522 \mathrm{~nm}$, with increasing solvent apolarity. Hence, the Stokes shift of the ester 11e and cyano 11d dyes are larger in a polar solvent than in an apolar solvent. This blue shift is most pronounced in the ester compound 11e, when going from acetonitrile to toluene the emission maxima display a hypsochromic shift of $62 \mathrm{~nm}$ for the cyano dye 11d and $79 \mathrm{~nm}$ for the ester dye 11e. Lastly, the emission peaks of both fluorophores in the polar acetonitrile are broader than their absorption peaks, whereas in the apolar toluene they are narrower. The broadness of these peaks for the cyano 11d and ester 11e compounds are similar to each other.

The nitrophenyl dye 11c behaves differently than the corresponding cyano 11d and ester 11e compounds. Most noticeably is that this triazapentalene with a strongly electron withdrawing nitro group at its 3-position is not fluorescent. In contrast, its 2substituted derivative, 2-(4-nitrophenyl)-triazapentalene, has been reported before to be fluorescent. ${ }^{2 a}$ Another difference with the other synthesized triazapentalenes is that the absorption maxima of the 3-(4-nitrophenyl) dye 11c are red shifted, by about $70 \mathrm{~nm}$, compared to its cyano 11d and ester 11e derivatives. Furthermore, the absorption peak of the nitro compound 11c is broader than those of the other two 3-aryl triazapentalenes. The nonfluorescence, the red shifted absorption and the broader peaks of nitrophenyl dye 11c, compared to the corresponding cyano 11d and ester 11e compounds, might be due to a photoinduced intramolecular charge transfer caused by the strongly electron withdrawing nitro group. ${ }^{1 \mathrm{a}}$

The fluorescence quantum yields of the two fluorescent 3aryl dyes were determined and proved to be higher, particularly in DCM, than the reference 2-(4-cyanophenyl)-triazapentalene 12. In fact, the synthesized cyano 11d and ester 11e fluorophores are both among the most fluorescent triazapentalenes described in the literature. ${ }^{2,3}$ To the best of our knowledge, the 3(4-(methoxycarbonyl)phenyl) dye 11e possesses the highest fluorescence quantum yield (0.79) of all the 1,3a,6atriazapentalene compounds reported so far.

In conclusion, using a metal-free three-component triazolization reaction novel 3-arylated 1,3a,6a-triazapentalenes were synthesized in two steps starting from simple commercial starting materials without needing a toxic heavy metal catalyst. This proved to be a good strategy to functionalize the 3-position of the triazapentalene core. Furthermore, introducing electron withdrawing groups onto this position was demonstrated to give stable triazapentalene dyes, which allowed their full spectroscopic characterization and is important to develop applications for these dyes. The resulting 3-(4-cyanophenyl) 11d and 3-(4(methoxycarbonyl)phenyl) 11e compounds were found to be highly fluorescent compounds. Moreover, both possess a reactive functional group, a cyano or an ester group, that might be useful as reactive handles for further functionalization.

\section{ASSOCIATED CONTENT}

\section{Supporting Information}

The Supporting Information is available free of charge on the ACS Publications website.

Experimental procedures and characterization data $\left({ }^{1} \mathrm{H}\right.$ and ${ }^{13} \mathrm{C}$ NMR spectra of all new compounds, NMR spectra and mass spectra of unstable triazapentalenes and absorption and emission spectra of the triazapentalene dyes) (PDF)

\section{AUTHOR INFORMATION}

\author{
Corresponding Author \\ *E-mail: Wim.Dehaen@kuleuven.be
}

\section{Author Contributions}

All authors have given approval to the final version of the manuscript.

\section{ACKNOWLEDGMENT}

KU Leuven is thanked for continued financial support, and for a postdoctoral fellowship to B.V. We thank Dr Joice Thomas (Department of Chemistry, KU Leuven) for help at the initial stages of this research (synthesis of 10a).

\section{REFERENCES}

(1) (a) Valeur, B. Molecular Fluorescence. Principles and Applications; Wiley-VCH: Weinheim, Germany, 2002. (b) Lakowicz, J. R. Principles of fluorescence spectroscopy, 3rd edn; Springer: New York, 2006. (c) Lavis, L. D.; Raines, R. T. ACS Chem. Biol. 2008, 3, 142 155 .

(2) (a) Namba, K.; Osawa, A.; Ishizaka, S.; Kitamuran N.; Tanino, K. J. Am. Chem. Soc. 2011, 133, 11466-11469. (b) Namba, K.; Mera, A.; Osawa, A.; Sakuda, E.; Kitamura, N.; Tanino, K. Org. Lett. 2012 , 14, 5554-5557. (c) Namba, K.; Osawa, A.; Nakayama, A.; Mera, A.; Tano, F.; Chuman, Y.; Sakuda, E.; Taketsugu, T.; Sakaguchi, K.; Kitamura, N.; Tanino, K. Chem. Sci. 2015, 6, 1083-1093. (d) Kamada, R.; Tano, F.; Kudoh, F.; Kimura, N.; Chuman, Y.; Osawa, A.; Namba, K.; Tanino, K.; Sakaguchi, K. PLoS ONE 2016, 11, e0160625. (e) Nakayama, A.; Nishio, S.; Otani, A.; Mera, A.; Osawa, A.; Tanino, K.; Namba, K. Chem. Pharm. Bull. 2016, 64, 830-837. (f) Sawada, J.; Osawa, A.; Takeuchi, T.; Kaneda, M.; Oishi, S.; Fujii, N.; Asai, A.; Tanino, K.; Namba K. Bioorg. Med. Chem. Lett. 2016, 26, 5765-5769.

(3) Cai, R.; Wang, D.; Chen, Y.; Yan, W.; Geise, N. R.; Sharma, S.; Li, H.; Petersen, J. L.; Li, M.; Shi, X. Chem. Commun. 2014, 50, 73037305.

(4) Koga, H.; Hirobe, M.; Okamoto, T. Tetrahedron Lett., 1978, 19 , 1291-1294.

(5) Trofimenko, S. J. Am. Chem. Soc. 1966, 88, 5588-5592.

(6) Knox, S. A. R.; Stone, F. G. A. Acc. Chem. Res. 1974, 7, 321328.

(7) Thomas, J.; Jana, S.; John, J.; Liekens, S.; Dehaen, W. Chem. Commun. 2016, 52, 2885-2888.

(8) (a) Su, T. M.; Sliwinski, W. F.; Schleyer, P. V. R. J. Am. Chem. Soc. 1969, 91, 5386-5388. (b) Beard, C. D.; Baum, K.; Grakauskas, V. J. Org. Chem. 1973, 38, 3673-3677. 